\title{
An adaptation of hydrophobic interaction chromatography for estimation of protein solubility optima ${ }^{1}$
}

\author{
Pete Gagnon $^{\mathrm{a}, *}$, Terry Mayes ${ }^{\mathrm{b}}$, Åke Danielsson ${ }^{\mathrm{c}}$ \\ a Validated Biosystems Incorporated, 5800 N. Kolb Road, Ste. 5127, Tucson, AZ 85750, USA \\ b Pharmacia Biotech, 800 Centennial Avenue, Piscataway, NJ 08854, USA \\ c Pharmacia Biotech, S-751 82 Uppsala, Sweden
}

Received 6 January 1997; received in revised form 31 March 1997

\begin{abstract}
This study describes and adaptation of hydrophobic interaction chromatography (HIC) that can be used to estimate protein solubility optima. The method does not support determination of absolute, e.g. quantitative solubility, however it does provide a basis for identifying the salt concentration, $\mathrm{pH}$, or additive concentrations that support the highest relative solubility. The magnitude of a given salt's effects are consistent with its ranking in the Hofmeister series. IgG in solutions of strong 'precipitating' salts exhibits a classical salting-in/salting-out curve, described by a solubility minimum at low ionic strength, increasing to a well-defined maximum, and then losing solubility with further elevation of salt concentration. The direct effect of $\mathrm{pH}$ on protein solubility, as well as its indirect effect via modification of the ionic equilibria of dissolved salts, can also be tracked. Cooperative effects of solubility modifiers such as amino acids can likewise be assessed. The technique can be a useful tool in the development of liquid formulations for protein pharmaceuticals. (C) 1997 Elsevier Science B.V.
\end{abstract}

Keywords: Formulation of protein pharmaceuticals; Estimation of protein solubility; Hydrophobic interaction chromatography; Effects of salts on protein solubility; Effects of $\mathrm{pH}$ on protein solubility; Hofmeister series

\section{Introduction}

Protein solubility and conformational stability are known to be sensitive functions of the chemical environments in which the proteins reside, and both are important parameters in protein formulation [1-4]. Unfortunately, the effects of a given

\footnotetext{
* Corresponding author. Tel. + 1520 5291095; fax: + 1520 5291021; e-mail: pete@validated.com

${ }^{1}$ Presented at the Well Characterized Biotechnology Pharmaceuticals Meeting in San Francisco, 6-8 January 1997.
}

chemical environment on stability do not necessarily parallel its effects on solubility. Chaotropic agents improve solubility, but do so at the expense of stability $[1,3]$. The obvious tactic would seem to be exclusive deployment of known stabilizing agents, but many of the most effective ones are also strong precipitants, or 'salting-out' agents, as is the case with salts high up in the Hofmeister lyotropic series [1,3-10]. Along with protein self-association, excessive concentrations of these agents can promote hetero-association with other surfaces. 
Protein solubility can provide a useful index for identifying the optimum concentrations for stabilizing additives and for other parameters such as $\mathrm{pH}$. Maximum solubility translates into minimum chemical potential, which in turn translates into the minimum tendency of a protein toward selfor hetero-association [1-4]. The challenge is to identify the conditions that support maximum solubility. Traditional techniques require evaluating the ability of various formulations to resolubilize supersaturating amounts of crystallized or lyophilized protein $[4,6]$. They are laborious, time consuming, and require large amounts of protein, not to mention the fact that lyophilization may itself cause aggregation or otherwise alter native protein solubility characteristics. These limitations are amplified by the need to evaluate large numbers of formulations during the course of development.

Another approach is to dialyse soluble purified protein against various buffer formulations, centrifuge or filter out any precipitate, then measure the amount of protein remaining in solution [7]. This requires less protein but is still laborious and has the disadvantage of leaving a 'blackout' zone: it doesn't allow discrimination of relative solubilization capacity among formulations in which the protein remains fully soluble.

A variant of HIC offers a third alternative. The parallel between protein precipitation and retention on hydrophobic chromatography supports has long been recognized [6,7]. Both phenomena have been related to protein chemical potential in a particular buffer or mobile phase [8-12]. Traditional HIC retention studies, with experiments evaluating the capacity factor, suffer from the same type of blackout zone noted above in conjunction with some solubility experiments. This makes it impossible to use HIC elution characteristics as an index. However, we have observed that the parallel between solubility and HIC behavior extends beyond retention behavior. Even under mobile phase conditions where proteincolumn interactions are too weak for a protein to be retained, transient interactions nevertheless occur. These retard progress of the protein as it passes through the column, broadening the protein 'zone', and proportionately reducing the height of the unretained (fall-through) peak. The taller the unretained peak, the less interaction between the protein and the column, which should reflect a parallel tendency away from selfor hetero-association in free solution. Unretained peak height should thereby provide an index of protein solubility.

In this study we report experimental results obtain with fluoresceinated and unlabeled monoclonal antibodies on chromatography supports representing a range of hydrophobicities. Sodium salts representing different rankings in the Hofmeister series were evaluated over a range of concentrations and $\mathrm{pH}$ values. Effects of glycine, which is reported to be an antibody solubilizing/ stabilizing agent but a precipitant of fibrinogen, were also evaluated [13-16]. Possible applications of the technique to formulation development, as well as its limitations, are discussed.

\section{Experimental}

Chromatography was conducted with an ÄKTA $^{\mathrm{TM}}$ explorer liquid chromatograph, on prepacked $1 \mathrm{ml}$ RESOURCE $^{\circledR}$ 15ETH, 15ISO and 15PHE columns (Pharmacia Biotech, Piscataway, NJ). The listed order of these media reflects their relative hydrophobicity, 15PHE being the most strongly hydrophobic. Detailed physicochemical description and comparative performance characterization are provided in reference [7].

Purified carboxy-fluoresceinated and unlabeled monoclonal antibodies were obtained from Becton Dickinson Immunocytometry Systems, San Jose, CA). American Chemical Society grade buffers and salts were purchased from Sigma (St. Louis, MO). Buffers were prepared with reverse osmosis-deionized water, vacuum filtered to 0.22 $\mu \mathrm{m}$, and assigned 5-day expirations. Buffer pairs were prepared, each with a foundation buffer containing $0.01 \mathrm{M}$ buffer. Morpholinoethanesulfonic acid (MES) was used for buffering at $\mathrm{pH}$ 5.0, hydroxyethylpiperazine ethanesulfonic acid (Hepes) at $\mathrm{pH} 7.0$, and trishydroxyaminomethane (Tris) at $\mathrm{pH}$ 9.0. these buffers were chosen for the combination of their strong buffer capacity with 
minimum contribution to conductivity. MES and Hepes buffers were titrated to $\mathrm{pH}$ with sodium hydroxide. Tris buffers were titrated with hydrochloric acid. The second member of each buffer pair contained the salt under study, at a concentration of $1.0 \mathrm{M}$. Experiments with glycine were conducted with $1.0 \mathrm{M}$ glycine in $0.01 \mathrm{M}$ Hepes, pH 7.0 as the foundation buffer, and its high salt counterpart the same except for inclusion of $1.0 \mathrm{M}$ sodium citrate.

All experiments were conducted at $21^{\circ} \mathrm{C}$ at a

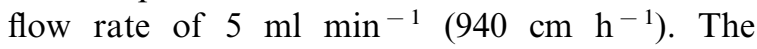
column was equilibrated with 10 column volumes (CV) of buffer, then injected with $20 \mu \mathrm{l}$ of purified antibody $(10 \mu \mathrm{g})$ in $0.05 \mathrm{M}$ sodium phosphate, 0.1 $\mathrm{M}$ sodium chloride, $\mathrm{pH}$ 7.0. All sample injections were programmed through an autoloader/autoinjector to ensure run-to-run precision. The column was then washed with $5 \mathrm{CV}$ equilibrating buffer, and eluted with $5 \mathrm{CV} 0.05 \mathrm{M}$ concentration of whatever salt was under study. Experiments were monitored at $280 \mathrm{~nm}$ UV. In each series, unretained peak height was measured in mobile phase formulations of $0.0,0.008,0.016,0.031,0.062$, $0.125,0.25,0.5$ and $1.0 \mathrm{M}$ salt. These increments were produced by programmed proportioning of buffer pairs through the chromatograph.

\section{Results and discussion}

Fig. 1 illustrates results from experiments with a carboxy-fluoresceinated $\operatorname{IgG}$ monoclonal in sodium sulfate at $\mathrm{pH} 7.0$ on the 15ISO column. The overall pattern reflects the classical protein salting-in/salting-out phenomenon, where increasing salt concentration initially improves solubility, then depresses it. The salting-in effect has been attributed to electrostatic interactions between the protein and dissolved ions $[9,11,12]$. The saltingout effect has been attributed to exclusion of dissolved ions from peptide bonds and hydrophobic residues [1-3]. This leaves the proteins preferentially hydrated and stabilized but creates a thermodynamically unfavorable discontinuity between the pure-water sheath surrounding the protein and the salt-containing solvent. The discontinuity favors association of the proteins,

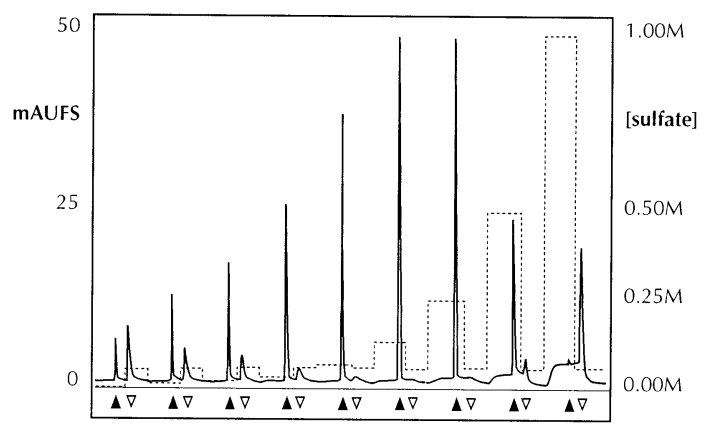

Fig. 1. Results from analysis of the effects of sodium sulfate concentration at $\mathrm{pH} 7.0$ on unretained peak height of a carboxy-fluoresceinated IgG monoclonal antibody on RESOURCE 15ISO. See Section 2 for conditions. The dark up-arrows mark the unretained peak. The white down-arrows mark the elution peak. The dotted line indicates the molarity of sodium sulfate.

among themselves and with other hydrated surfaces, by cohydration/cosolvent exclusion $[1,3,8]$.

Fig. 2 shows that the balance between salting-in and salting-out is a function of salt identity as well as concentration. Precipitating salts like sodium sulfate, phosphate and citrate reveal welldefined solubilizing optima, the relative concentrations of which are consistent with their rankings in the Hofmeister series $[1,3,8,9]$. Chaotropic salts like sodium perchlorate reveal a different pattern. Chaotropic ions are able to

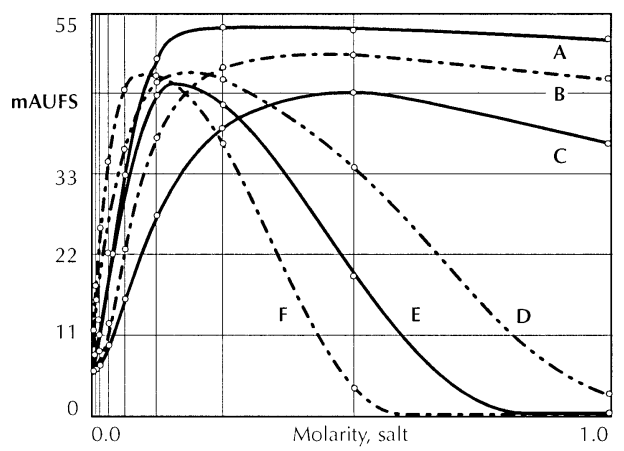

Fig. 2. The effects of multiple sodium salts at $\mathrm{pH} 7.0$ on unretained peak height of a carboxy-fluoresceinated IgG monoclonal antibody on RESOURCE 15ISO. See Section 2 for conditions. A, sodium perchlorate; B, sodium chloride; C, sodium acetate; D, sodium phosphate; E, sodium sulfate; F, sodium citrate. 


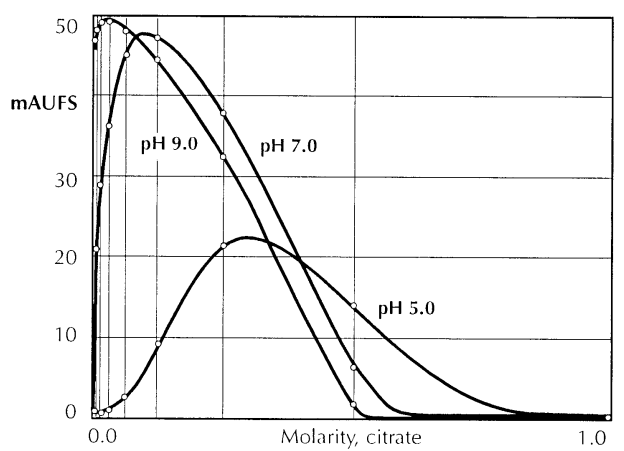

Fig. 3. The effects of $\mathrm{pH}$ over a range of sodium citrate concentrations on unretained peak height of a carboxy-fluoresceinated IgG monoclonal antibody on RESOURCE 15ISO. See Section 2 for conditions.

penetrate a protein's hydration sheath and bind directly to peptide bonds and hydrophobic residues. This increases the net charge on the protein, increasing its interactivity with the polar solvent, and reducing its chemical potential relative to the solvent $[1,3]$. This maximizes solubility; producing a broad concentration zone across which solubility is largely independent of salt concentration. However, it does so at the expense of stability; loss of local hydrophobicity due to chaotropic ion-binding disrupts the protein's native architecture.

Fig. 3 illustrates the compound effects of $\mathrm{pH}$ as a function of sodium citrate concentration. The isoelectric point $(\mathrm{pl})$ of this antibody was polydisperse, ranging from about 5.3 to 5.8. As expected, unretained peak heights were smallest at the $\mathrm{pH}$ nearest the protein's pl. We attributed the $\mathrm{pH}$-dependent lateral shift of the profiles to alteration of citrate's salting-out capability, coincident with its titration state. Its second and third $\mathrm{p} K_{\mathrm{a}} \mathrm{s}$ are at $\mathrm{pH}$ 4.76 and 6.40 [17]. No such lateral shift was observed with sodium sulfate (data not shown). Although citrate itself is not a common formulation component, phosphate and other agents also have $\mathrm{p} K_{\mathrm{a}} \mathrm{s}$ within the $\mathrm{pH}$ range normally scouted in development. This highlights the importance of experimentally evaluating compound formulation effects.

Fig. 4 illustrates the comparison of results obtained from sodium citrate at $\mathrm{pH}$ 7.0, with those obtained from sodium citrate plus $1.0 \mathrm{M}$ glycine at the same $\mathrm{pH}$. Like precipitating salts, glycine is excluded from protein surfaces, yielding a net stabilizing effect [2]. Consistent with expectations, this also results in its enhancement of salt-mediated effects. This is observed as the protein solubility optimum being shifted to a lower salt concentration. These data point out the ability of the technique to discriminate and optimize the effects of additives in a base formulation. As with $\mathrm{pH}$, the data also emphasize the importance of evaluating compound formulations experimentally; simply adding a blind concentration of a reputed solubilizer to an already optimized formulation may promote unwanted secondary effects.

The glycine data are also important because they draw attention to a source of error in the method. Note that the amplitude of the optimum value in glycine is lower than the optimum in its absence. Rather than indicating that glycine has an overall suppressive effect on solubility, this most likely reflects the higher viscosity of the combined salt-glycine solutions. This is to be expected when dealing with diffusional limitations of porous chromatography supports, since diffusivity declines in proportion with viscosity [18]. This suggests that flow rate should affect unretained peak height, which is indeed the case. The slower the flow rate, the higher the peak (data not shown). These factors do not confound identifica-

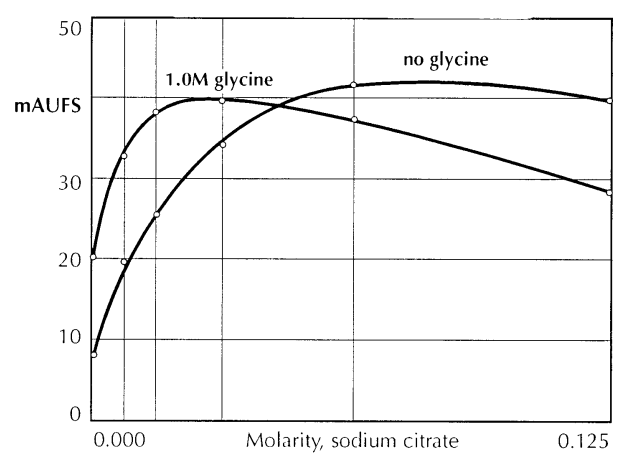

Fig. 4. Alteration of solubility optima by addition of glycine. Unretained peak height of an $\mathrm{IgG}$ monoclonal antibody on RESOURCE 15ETH, 15ISO, 15PHE. Sodium citrate at $\mathrm{pH}$ 7.0. See Section 2 for conditions. 


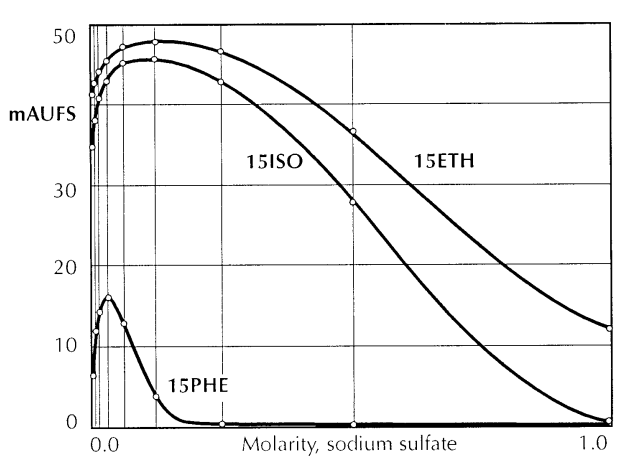

Fig. 5. The effect of column hydrophobicity on unretained peak height of an IgG monoclonal antibody on RESOURCE 15ETH, 15ISO and 15PHE. Sodium citrate at $\mathrm{pH}$ 7.0. See Section 2 for conditions.

tion of the optimum solubility conditions within a set of properly controlled experiments, but they do emphasize the importance of conducting all the experiments in a set under identical conditions, and they recommend caution with respect to drawing conclusions about the relative effects among different experimental treatments, based on curve amplitudes.

A potentially more serious issue concerns the contribution of column hydrophobicity to the results. Hydrophobic interactions are a strong attractive force in salt solutions, exerting up to 2 orders of magnitude greater influence than van der Waals forces at distances up to $8 \mathrm{~nm}$ [19]. In addition, proteins orient themselves so as to present their most hydrophobic surface to the ligand [20,21]. The associative properties of the adsorptive surface are therefore likely to be different from the free-solution solubility properties of the protein as a whole. The question is - to what extent?

Lacking sufficient protein to conduct traditional solubility studies, we compared results among chromatographic supports with different hydrophobicities. Since the strength of a protein's interaction is proportional to the hydrophobicity of the adsorptive surface, the nature and magnitude of differences among the columns should reveal the extent to which results can be relied upon to reflect protein solubility in free solution $[8,19]$. Fig. 5 compares results obtained with mon- oclonal IgG on the 3 columns. The salt concentration skew of the 'solubility' maximum on the 15PHE column, relative to the 2 less hydrophobic columns, warns that strongly hydrophobic columns confound correlation of HIC data with solubility. At the same time, the disparity in the amplitude of the response curves for the 2 weaker columns highlight the unsuitability of the data for evaluating quantitative solubility. Nevertheless, the conformity of the concentration optima for the 2 weaker columns indicates that this method can be used reliably to assess relative solubility. Additional work may reveal experimental methods and formulae that make it possible to factor out the hydrophobic contribution of various column media and derive quantitative information, but for the present, the technique should be limited to weakly hydrophobic columns, and with the tacit understanding that it be used only to define relative solubility optima - not quantitative solubility.

\section{Conclusions}

Evaluation of unretained protein peak height on weakly hydrophobic chromatography columns offers a compelling parallel with protein solubility behavior. Whereas quantitative solubility data is time consuming and expensive to obtain, the present adaptation of HIC is rapid and economical. Equipment for performing the technique is common to most protein chemistry laboratories, and it can be readily automated to achieve high through-put on chromatographs able to accommodate preprogrammed templates.

The primary value of the technique resides not in its ability to replace other formulation development tools, but to augment them. Most of the current methodologies are directed exclusively toward measuring protein stability. Stability is undeniably critical, but protein-protein and other protein-surface associations caused by excessive concentrations of stabilizing agents may interfere with the product application. Evaluating unretained peak height by HIC provides a systematic and objective basis for balancing the solubilizing and stabilizing influences of protein formulation 
components. Especially as a scouting tool, this method can help focus more detailed investigations on the most promising formulary components and compositions, thereby accelerating the overall task of formulation development.

\section{References}

[1] T. Arakawa, S. Timasheff, Biochemistry 21 (1982) 65456552.

[2] T. Arakawa, S. Timasheff, Arch. Biochem. Biophys. 224 (1983) $169-177$

[3] T. Arakawa, S. Timasheff, Biochemistry 23 (1984) 5914 5923.

[4] M. Dixon, E. Webb, Adv. Protein Chem. 16 (1961) $197-219$.

[5] F. Hofmeister, Arch. Exp. Pathol. Pharmakol. 24 (1888) $247-263$

[6] A. Green, J. Biol. Chem. 93 (1931) 495-516.

[7] P. Gagnon, E. Grund, T. Lindbäck, BioPharm. 8 (3) (1995) 21-27.

[8] B. Roetger, J. Myers, M. Ladisch, F. Regnier, ACS Symposium Series \# 427, American Chemical Society, Washington DC, 1990, pp. 81-92.
[9] W. Melander, C. Horvath, Arch. Biochem. Biophys. 183 (1977) 200-215.

[10] R. Melander, C. Corradini, C. Horvath, J. Chromatogr. 317 (1984) 67-85.

[11] E. Cohn, J. Edsall, Proteins, Amino Acids, and Peptides as Ions and Dipolar Ions, Rheinhold, New York, 1969.

[12] P. von Hippel, T. Schleich, Structure and stability of biological macromolecules, in: S. Timasheff, G. Fasman (Eds.), Marcel Dekker, New York, 1969, pp. 417-574.

[13] J. Porath, N. Ui, Biochim. Biophys. Acta 90 (1964) 324.

[14] L. Kazal, C. Amsell, O. Miller, L. Tocantis, Proc. Exp. Biol. Med. 113 (1963) 989.

[15] U.-B. Hanson, E. Nilsson, J. Immunol. Methods 2 (1973) 221.

[16] E. Kabat, M. Mayer, Experimental Immunochemistry, 2nd ed., Thomas, Springfield, 1966, p. 264.

[17] D. Perrin, B. Dempsey, Buffers for $\mathrm{pH}$ and Metal Ion Control, Chapman and Hall, New York, 1974.

[18] L. Hagel, Protein Purification: Principles, High Resolution Methods, and Applications, in: J.-C. Janson, L. Ryden (Eds.), VCH, New York, 1989, p. 63.

[19] C. Helm, J. Iraelachvilli, P. McGuigan, Science 246 (1989) 919-922.

[20] J. Fausnaugh, F. Regnier, J. Chromatogr. 359 (1986) $131-146$.

[21] R. Chicz, F. Regnier, J. Chromatogr. 500 (1990) 503518. 